\title{
Inconsistency of Timeliness in a Chief Complaint-Based Syndromic Surveillance System During Two Influenza Epidemic Seasons
}

\author{
Tao Tao*1, Qi Zhao', Jun Zong ${ }^{2}$, Xue Li ${ }^{3}$, Vinod Diwan ${ }^{4}$ and Biao Xu \\ ${ }^{1}$ School of Public Health, Fudan University, Shanghai, China; ${ }^{2}$ Jiangxi CDC, Nanchang, China; ${ }^{3}$ Future Position X, Gävle, Sweden; \\ ${ }^{4}$ Department of Public Health Sciences, Karolinska Institutet, Stockholm, Sweden
}

\section{Objective}

To study and compare the timeliness of syndromic surveillance system for the early warning of infectious diseases among different epidemic seasons.

\section{Introduction}

Syndromic surveillance system has been developed and implemented all over the world, and many studies showed that syndromic data sources had improved timeliness towards traditional surveillance method in the early warning of some infectious disease epidemics. However, owing to the uncertainties of disease epidemic features, clinical manifestations and population behaviors, the early warning timeliness of syndromic data sources might change across time and population, and few studies had explored their consistency in different epidemic periods of infectious diseases.

\section{Methods}

We evaluated the timeliness of a chief complaint-based syndromic surveillance system established in two rural counties of Jiangxi Province, China, towards seasonal influenza epidemics. Weekly number of influenza-like illness (ILI) patients counted from syndromic surveillance system was compared with the weekly influenza virus positive rate (VPR) data collected from National Influenza Surveillance Network in Jiangxi Province using cross correlation method. Timeliness was defined as the lead weeks of ILI data towards VPR data when two data sources reached maximum correlation. Daily data check and quarterly field data quality control was conducted to ensure the correctness and completeness of data in syndromic surveillance system.

\section{Results}

From $18^{\text {th }}$ week 2012 to $52^{\text {nd }}$ week 2013 , there were two influenza epidemic seasons in surveillance areas according to the VPR data from National Influenza Surveillance Network. Apparent correlation could be observed between ILI and VPR curves, and in some periods the ILI peaks occurred earlier than VPR peaks (Figure 1). Cross correlation study showed that in Epidemic Season 1, the subgroups of ILI data had 0 5 weeks'timeliness towards VPR data, with correlation coefficients between 0.52 0.84. ILI reported from upper-level health facilities (county and township hospitals), younger patient groups (3-17 years) had higher timeliness than ILI reported from village health stations, elder patient groups (18-years) (Table 1). The situation in Epidemic Season 2 were quite different that the correlations between ILI subgroups and VPR data were generally weaker than that in Epidemic Season 1, and some ILI subgroups even presented 1 5 weeks'delays towards VPR data. ILI reported from village health station, elder patient groups (18- years) had better timeliness than that from upper-level health facilities, younger patient groups (0-17 years) (Table 2). Lab data showed that the dominant influenza strains were different in two epidemic seasons.

\section{Conclusions}

The inconsistency of timeliness in syndromic surveillance system might be attributed to the differences in dominant strains, clinical manifestations of influenza, population age groups affected, and health seeking behaviors of influenza patients in two epidemic seasons. Further exploring the causes of these phenomena could help public health professionals select most timely data sources for the early warning of influenza epidemics in rural China.
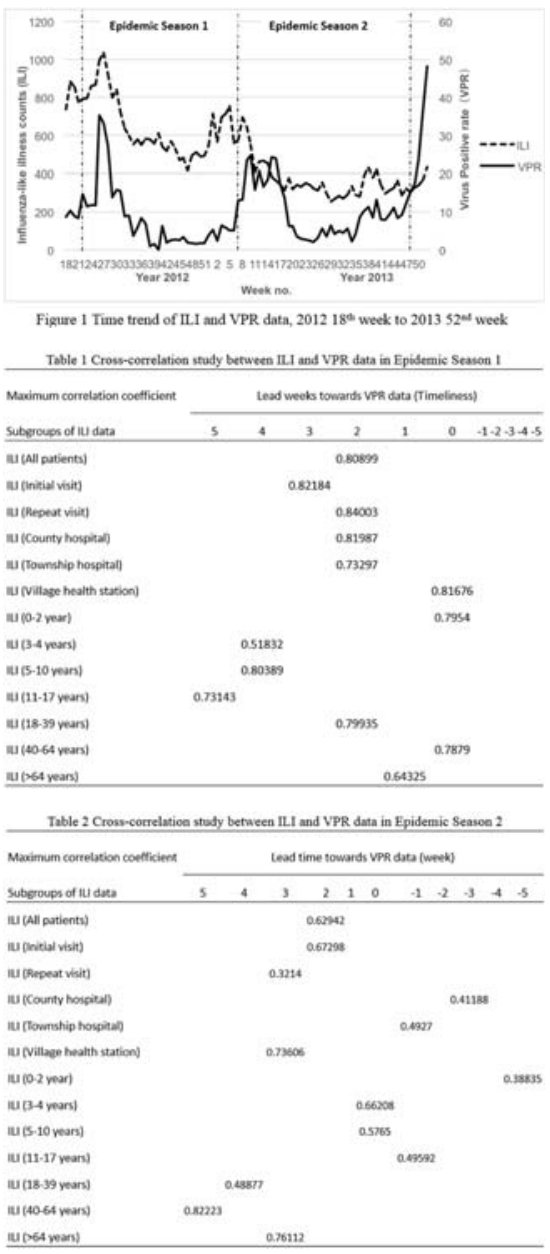

Keywords

Timeliness; Syndromic surveillance; Epidemic season

*Tao Tao

E-mail: ttsuper2000@hotmail.com 